\title{
An animal-specific FSI model of the abdominal aorta in anesthetized mice
}

\author{
Bram Trachet $^{1,3^{*}}$, Joris Bols ${ }^{1,2^{*}}$, Joris Degroote ${ }^{2}$, Benedict Verhegghe ${ }^{1}$, Nikolaos \\ Stergiopulos $^{3}$, Jan Vierendeels ${ }^{2}$, Patrick Segers ${ }^{1}$ \\ ${ }^{1}$ IBiTech-bioMMeda, Ghent University - iMinds Medical IT, Ghent, BE \\ ${ }^{2}$ Department of Flow, Heat and Combustion Mechanics, Ghent University, Ghent, BE \\ ${ }^{3}$ Institute of Bioengineering, EPFL, Lausanne, $\mathrm{CH}$
}

*both authors contributed equally to this work

Corresponding author:

Bram Trachet

De Pintelaan 185B

9000 Ghent

Bram. Trachet@ugent.be 
This is a pre-copyedited, author-produced PDF of an article accepted for publication in Annals of Biomedical Engineering following peer review. The version of record is available online at:

An Animal-Specific FSI Model of the Abdominal Aorta in Anesthetized Mice

B Trachet, J Bols, J Degroote, B Verhegghe, N Stergiopulos, J Vierendeels, P Segers

Annals of Biomedical Engineering 2015

doi: http://link.springer.com/article/10.1007/s10439-015-1310-y 


\section{Abstract}

Recent research has revealed that angiotensin II-induced abdominal aortic aneurysm in mice can be related to medial ruptures occurring in the vicinity of abdominal side branches. Nevertheless a thorough understanding of the biomechanics near abdominal side branches in mice is lacking. In the current work we present a mouse-specific fluid-structure interaction (FSI) model of the abdominal aorta in $\mathrm{ApoE}^{-/-}$mice that incorporates in vivo stresses. The aortic geometry was based on contrast-enhanced in vivo micro-CT images, while aortic flow boundary conditions and material model parameters were based on in vivo high-frequency ultrasound. Flow waveforms predicted by FSI simulations corresponded better to in vivo measurements than those from CFD simulations. Peak-systolic principal stresses at the inner and outer aortic wall were locally increased caudal to the celiac and left lateral to the celiac and mesenteric arteries. Interestingly, these were also the locations at which a tear in the tunica media had been observed in previous work on angiotensin II-infused mice. Our preliminary results therefore suggest that local biomechanics play an important role in the pathophysiology of branch-related ruptures in angiotensin-II infused mice. More elaborate follow-up research is needed to demonstrate the role of biomechanics and mechanobiology in a longitudinal setting.

Key-words: fluid-structure interaction, computational fluid dynamics, abdominal aorta, mouse model, mouse-specific, high-frequency ultrasound, micro-CT, abdominal aortic aneurysm, dissecting aneurysm 


\section{Introduction}

Mouse models are often used to study the onset and progression of cardiovascular disease due to the availability of different genetically modified strains and the rapid time course of disease development. The angiotensin II-infused $\mathrm{ApoE}^{-/-}$mouse model in particular has long been an established pre-clinical model for abdominal aortic aneurysm, since murine aneurysms - like their human counterparts - are associated with luminal dilatation, macrophage infiltration, medial elastolysis and thrombus formation[1, 2]. On the other hand, recent insights challenge the relevance of this particular mouse model to study human aneurysms pathophysiology, and indicate that aortic dissection plays a key role in the induced aortic disease in these animals. Gavish et al. were the first to hypothesize that abdominal side branches play a pivotal role in the pathophysiology of dissecting aortic aneurysm in these animals [3]. We recently used a novel post mortem imaging technique called PCXTM (phase contrast X-ray tomographic microscopy) and could indeed confirm this hypothesis. Our high-resolution post mortem images showed that the intramural hematoma was related to ruptures in the tunica media near suprarenal side branches (such as intercostals and the superior suprarenal artery). Moreover, apparent luminal dilatation could be linked to a tear in the tunica media that appeared caudal, cranial or left lateral to the celiac artery [4].

However, the biomechanics and mechanobiology underlying these branch-related ruptures in this mouse model still remains to be demonstrated. Fluid dynamics might play an important role as the flow field and wall shear stress might be disturbed near branch bifurcations, which in turn might lead to endothelial dysfunction [5,6]. Solid mechanics could provide another approach to explain branch-related ruptures as the mechanical tension exerted on the tunica media is expected to be elevated near branch bifurcations. Both CFD (Computational Fluid 
Dynamics to simulate the flow field in a model with rigid walls) and CSM (Computational Solid Mechanics to simulate the wall stress without accounting for the fluid domain) have been used to study arterial fluid dynamics [5-13] and solid mechanics [14-16] in normal [7-12, 1416] and aneurysmatic $[5,6,13]$ mice. However, the appropriate pressure and shear distribution as a function of time are essential inputs for a correct CSM simulation, while the inclusion of distending walls has been shown to significantly influence the calculated flow field with CFD [17]. Therefore fluid-structure interaction (FSI) simulations, which account for both the fluid mechanics and structural mechanics problem simultaneously, are increasingly performed on a patient-specific basis[18-22]. Nevertheless, mouse-specific FSI simulations have (to the best of our knowledge) never been performed. The required inputs for such computational methods (i.e. a mouse-specific geometry and boundary conditions for both the fluid and solid domain) are often difficult to obtain, since the small size and rapid heart rate of these laboratory animals put high constraints on in vivo imaging tools. The scarce available literature focuses on either CFD or CSM, while a thorough understanding of the biomechanics in the murine aorta, and in particular the biomechanics of abdominal side branches, is still lacking.

The aim of the current work was twofold. First, we aimed to present a mouse-specific FSI simulation framework, based on in vivo measurements, in which fluid and solid mechanics are simultaneously accounted for in the murine abdominal aorta. Second, we applied this FSI approach to investigate how the distribution of locally decreased wall shear stress and locally increased maximum principal stress in the vicinity of abdominal side branches relates to the distribution of medial ruptures that had previously been observed in angiotensin II-infused mice.

\section{Methods}


Mice.

In vivo measurements were obtained from an in house bred ApoE ${ }^{-/}$mouse on a C57Bl/6 background (age: 37 weeks, body weight $30.6 \mathrm{~g}$ ) that was previously used in another study [11]. All procedures were approved by the Ethical Committee of Ghent University and performed according to the guidelines from Directive 2010/63/EU of the European Parliament on the protection of animals used for scientific purposes. All surgery and measurements were performed under $1.5 \%$ isoflurane anesthesia, all efforts were made to minimize suffering, and the 3R (refine, reduce, replace) principle was applied to minimize the number of laboratory animals.

\section{Micro-CT.}

The animal was scanned in dorsal recumbency using a micro-CT scanner dedicated for small animal imaging (GE FLEX Triumph, Gamma Medica-Ideas). Acquisition was performed with a $70 \mathrm{kVp}$ tube voltage and a current of $180 \mu \mathrm{A}$. Prior to scanning, the mouse was intravenously injected with 150 microliter/25 g body weight of Aurovist (Nanoprobes) to allow for a better discrimination between blood vessels and surrounding soft tissues. The scan lasted for 4.5 minutes, and images were obtained with a $33.81 \mathrm{~mm}$ transverse field of view. The continuous data were binned into 1024 projections and reconstructed with a proprietary software (Cobra EXXIM, EXXIM Computing corp., Livermore, USA) using a Feldkamp-type algorithm with Parker's weighting function in a 512 x 512 x 512 matrix with a $75 \mathrm{~mm}$ voxel size.

\section{High-frequency ultrasound.}

Ultrasound data were obtained with a high-frequency ultrasound apparatus (Vevo 2100, VisualSonics). All measurements were performed by an experienced operator (BT). The animal 
was secured on the table in dorsal recumbency while heart rate, respiratory rate and body temperature were monitored. Pulsed Doppler was used to assess the flow velocities in the abdominal aorta and in its side branches. Waveforms were traced within a custom-made environment platform in Matlab (Mathworks). For each measurement location, the average of three different waveforms was calculated. Measured flow velocities were treated as the maximum velocity component of a parabolic flow profile. Flow velocities were converted to volumetric flows using the corresponding cross-sectional area that was obtained from $3 \mathrm{D}$, segmented micro-CT data. The obtained flow waveforms corresponded to an average heart rate of 360 beats per minute and a cardiac cycle of $0.17 \mathrm{~s}$. To obtain a time-averaged mass imbalance equal to zero, the thoracic inlet flow was scaled with an imbalance correction factor of 1.07. We justify this assumption by the fact that it is more likely to underestimate the velocity measured by ultrasound than to overestimate it. Moreover, scaling the inflow required only one factor and minimized this factor. RF (radio frequency) diameter waveforms were obtained with long axis MMode imaging in the abdominal aorta, cranial to the celiac artery. A custom-written platform in Matlab, based on the algorithm described by Rabben et al [23], was used to reconstruct the complete MMode image from RF data. For each measurement, 3 cardiac cycles were subsequently selected and plotted as a reference for semi-automatic wall segmentation guided by the user. The tracked aortic wall movement yielded a highly accurate in vivo diameter waveform.

\section{Geometrical model of the abdominal aorta}

The reconstructed images were semi-automatically segmented using the software package Mimics (Materialise). Although contrast-enhanced images were used, manual intervention was 
still required to separate the aorta from the venous segments. After segmentation, the outer surface of the lumen geometry was represented by a triangulated surface mesh and exported into the stereolithographic (STL) file format. This geometry file was subsequently passed on to the extended Treemesh method, a parametric interface that has been implemented in pyFormex to generate hexahedral meshes[24]. Finally, a computational grid for the arterial wall was generated using quadratic hexahedral elements with a hybrid formulation. The thickness of the arterial wall was assumed to be $10 \%$ of the inner diameter. Several grids were auto-generated to ensure grid independency of the simulation results.

\section{Fluid dynamics model.}

All computational fluid dynamics (CFD) simulations have been performed with the commercial finite volume solver Fluent 14.5 (Ansys). Time-varying uniform velocity profiles were imposed normal to the boundaries at the inlet of the aorta (TA) and the outlets of the side branches (CA, RRA, MA, RLA), based on the flow velocity waveforms that had been obtained from in vivo measurements. Since these measurements were not taken at the exact same locations as the ending cross sections of our fluid domain, the difference in cross sectional area was accounted for. A pressure outlet boundary condition was prescribed at the distal abdominal aorta (AA) by means of a three element windkessel model which applies a static pressure at the outlet, $\mathrm{P}_{\mathrm{AA}}(\mathrm{t})$, given the flow through the outlet, $\mathrm{Q}_{\mathrm{AA}}(\mathrm{t})$. In the corresponding electrical analog circuit $\mathrm{Z}$ represents the characteristic impedance, $\mathrm{R}$ the hydraulic resistance and $\mathrm{C}$ the volume compliance (Fig. 1a) [25]. The parameters of the windkessel model were defined such that physiological pressure variations were retrieved when applying the measured flow rate at the distal abdominal aorta. $\left(\mathrm{Z}=367.1 \mathrm{mmHg} / \mathrm{ml} / \mathrm{s}, \mathrm{R}=2390.8 \mathrm{mmHg} / \mathrm{ml} / \mathrm{s}, \mathrm{C}=6.910^{-5} \mathrm{ml} / \mathrm{mmHg}\right.$ ). At the walls the no-slip condition was imposed. Regarding the fluid parameters, the density was set to $1060 \mathrm{~kg} / \mathrm{m}^{3}[7,8,26]$ and the blood was modeled as a Newtonian fluid with a constant 
dynamic viscosity of $0.0035 \mathrm{~Pa} \mathrm{~s}[7,8,27]$. The pressure and velocity fields were solved using the SIMPLE algorithm with second-order upwind discretization for the momentum equations and standard pressure interpolation (i.e. according to the momentum equation coefficients). An implicit time integration scheme was used with second-order accurate temporal discretization. Convergence was obtained when the scaled residuals (i.e. the residuals scaled relative to the largest absolute value of the residuals obtained in the first five iterations) of continuity and momentum decreased below $10^{-6}$.

\section{Structural mechanics model.}

All computational structural mechanics (CSM) simulations have been performed with the commercial finite element solver Abaqus Standard (Simulia). Transient simulations made use of an implicit (backward-Euler) time integration scheme, and geometric nonlinearities due to large deformations were taken into account. Local cylindrical coordinate systems were defined at the in- and outlets. At the corresponding ending cross sections, displacement boundary conditions were applied allowing radial displacement of the nodes only, except for the shortest side branch (left renal artery) where in plane movement of the nodes was allowed as well. Loads were only imposed at the inner surface of the vessel wall. In the FSI simulations, both the normal component and the shear component of the traction vector were applied at the fluidstructure interface. The aortic tissue was modeled using an Arruda-Boyce material model[28]. Details on how mouse-specific material constants were obtained are found below.

\section{Backward displacement method for zero stress geometry}

The in vivo stress field corresponding to the in vivo micro-CT images was computed based on the in house developed BDM (backward displacement method)[14]. The method computes the zero-pressure geometry iteratively by subtracting nodal displacements from the corresponding coordinates of the in vivo geometry. These nodal displacements are the result of a forward 
structural simulation in which an approximation of the zero-pressure geometry is inflated by the in vivo diastolic blood pressure which was taken $60 \mathrm{mmHg}$ [29]. Twenty-nine iterations were required to obtain a relative residual below $10^{-3}$. This corresponded to a mean distance of $2.510^{-4} \mathrm{~mm}$ and a maximum distance of $1.1910^{-3} \mathrm{~mm}$ which was still present between the nodes of the image-based geometry and the nodes of the geometry resulting from the forward problem. The resulting zero-pressure geometry is shown in Figure 1b. This geometry was subsequently inflated to the in vivo pressure to obtain the stress field corresponding to the in vivo geometry (at end-diastole).

\section{Backward displacement method for material parameter optimization}

In order to obtain material parameters that mimic the measured distension (eq. 1) of the arterial wall after inclusion of the in vivo stress field, we implemented a BDM-based material parameter optimization framework (BDMPO, Fig.1c). An objective function (eq. 2) was minimized using a standard minimization function in Matlab, with $d_{m}$ and $d_{s}$ the measured and simulated distensions. The optimization function utilized a Sequential Quadratic Programming (SQP) method with a BFGS-update of the Hessian in each iteration. In each iteration the backward displacement method was performed based on the in vivo geometry $\mathrm{x}_{\mathrm{m}}$, the internal pressure load $\mathrm{p}_{\mathrm{m}}$, and the new guess for the material parameters. Forward CSM computations were performed to bring the zero pressure geometry in its end-diastolic state $\left(\mathrm{P}_{\mathrm{dia}}\right)$, from which it was further inflated to peak-systole $\left(\mathrm{P}_{\mathrm{sys}}\right)$. Finally, the simulated distension $\mathrm{d}_{\mathrm{s}}$ was obtained (eq. 1) and compared to the measured $\mathrm{d}_{\mathrm{m}}$, (eq. 2). Upon convergence of the optimization procedure, the material parameters which result in the desired distension were obtained when applying the pulse pressure in a model in which the diastolic pressure is in equilibrium with the image-based and pre-stressed configuration. The BDMPO procedure was performed prior to the calculation of the zero stress geometry on a simplified model of a thick-walled cylindrical tube with an 
inner diameter of $0.32 \mathrm{~mm}$, which corresponds to the image-based diameter at the outlet of the distal abdominal aorta. Values of $\mathrm{d}_{\mathrm{m}}=10 \%$ (measured using RF MMode ultrasound), $\mathrm{P}_{\text {dia }}=60 \mathrm{mmHg}, \mathrm{P}_{\text {sys }}=90 \mathrm{mmHg}$ (literature values for anesthetized mice [29]) were used. In order to avoid over-parameterization of the problem, the aortic wall was modeled with the ArrudaBoyce material model (eq. 3,4). In these equations $\mathrm{W}$ is the strain-energy function, $\mathrm{I}_{1}$ the first invariant of the Cauchy-Green tensor, $\mu$ the shear modulus, $\mu_{0}$ the initial shear modulus and $\lambda_{\mathrm{m}}$ the locking stretch, which approximately denotes the stretch at which the slope of the stressstrain curve will rise significantly. The initial shear modulus is linked to the locking stretch by equation (5). The model can be seen as a fifth-order reduced polynomial, in which the five coefficients $\left(\mathrm{C}_{10}, \ldots, \mathrm{C}_{50}\right)$ are nonlinear functions of only two parameters $\mu$ and $\lambda_{\mathrm{m}}$. To provide sufficient stiffening in the physiological pressure range, a value of $\lambda_{\mathrm{m}}=1.01$ was considered. The BDMPO procedure was then performed to find the unknown material parameter $\mu=24358$ Pa.

$$
\begin{aligned}
& d=\frac{D_{s y s}-D_{d i a}}{D_{d i a}} \\
& f(\psi)=\left(\frac{d_{s}-d_{m}}{d_{m}}\right)^{2} \\
& W=\mu \sum_{i=1}^{5} \frac{\alpha_{i}}{\lambda_{m}^{2 i-2}}\left(I_{1}^{i}-3^{i}\right) \\
& \alpha_{1}=\frac{1}{2}, \alpha_{2}=\frac{1}{20}, \alpha_{3}=\frac{11}{1050}, \alpha_{4}=\frac{19}{7000}, \alpha_{5}=\frac{519}{67375 \lambda_{m}^{8}} \\
& \mu_{0}=\mu\left(1+\frac{3}{5 \lambda_{m}^{2}}+\frac{99}{175 \lambda_{m}^{4}}+\frac{513}{875 \lambda_{m}^{6}}+\frac{42039}{67375 \lambda_{m}^{8}}\right)
\end{aligned}
$$

\section{Coupling code.}

The flow solver (Fluent, Ansys) was coupled with the structural solver (Abaqus/Standard, Simulia) in a partitioned fluid-structure interaction (FSI) approach using the coupling code Tango[30]. This implies that the flow problem was solved for a given interface position, while 
the structural problem was solved for a stress boundary condition applied on the fluid-structure interface. The interface position, computed by the structural solver, was passed on to an IQNILS algorithm which provided a new prediction of the interface displacement and position used by the flow solver. Coupling iterations were performed between the flow solver and the structural solver in each time step to meet the equilibrium at the interface. The IQN-ILS algorithm is a quasi-Newton algorithm in which the inverse of the Jacobian is approximated by a least-squares model[30]. The interior fluid grid nodes were moved according to a spring analogy, with a spring constant inversely proportional to the edge length. This resulted in a smooth redistribution of the nodes in the fluid domain. To discretize the flow equations on a moving grid at the fluid-structure interface, the arbitrary Lagrangian-Eulerian (ALE) method was used. The FSI code has successfully been validated against other FSI codes in several benchmark cases [31]. A grid sensitivity analysis was performed for 7 different CFD cases with mesh densities increasing from 53k (coarse) to $3216 \mathrm{k}$ (fine, reference grid). Studied variables were (i) the pressure drop $\Delta \mathrm{p}$ along the centerline, (ii) the difference $\Delta \mathrm{v}$ between the maximum and minimum velocity along the centerline and (iii) the surface area enclosed by a WSS isocontour $\mathrm{A}_{\mathrm{iso}-w s s}$, that was evaluated at $10.8 \mathrm{~Pa}$. For each mesh, the relative error of these 3 variables was calculated with respect to the reference value that was obtained from the case with the finest grid. We found that $53 \mathrm{k}, 98 \mathrm{k}$ and $798 \mathrm{k}$ cells were sufficient to obtain a maximum error smaller than $1 \%$ for $\Delta \mathrm{p}, \mathrm{A}_{\text {iso-wss }}$ and $\Delta \mathrm{v}$ respectively. As a compromise between accuracy and computation time, a fluid grid of $156 \mathrm{k}$ cells and a solid domain of $44 \mathrm{k}$ quadratic elements were selected for the FSI simulation, which corresponded to an error (relative to the values resulting from the finest grid) of $0.3 \%$ for $\Delta \mathrm{p}, 0.8 \%$ for $\mathrm{A}_{\text {iso-wss, }}$ and $1.9 \%$ for $\Delta \mathrm{v}$.. The absolute convergence criterions for the coupling iterations were set to $10^{-6} \mathrm{~m}$ for the (L2-norm) residual of the interface displacement and to $5 \mathrm{~Pa}$ for the residual of the interface load. This resulted in 
an average of 5 coupling iterations per time step. A time step of $1.67 \mathrm{~ms}$ was used, which resulted in a computation time of $3 \mathrm{~h} 33$ min per cardiac cycle when performed on a Dell PowerEdge R620 server with two ten-core Intel Xeon E5-2680v2 CPUs at 2.8 GHz. In order to overcome startup phenomena, 4 cardiac cycles were simulated and all presented results were retained from the $4^{\text {th }}$ cycle. The difference in pulse pressure between the third and fourth cycle was less than $0.02 \%$. 


\section{Results}

CFD and FSI simulations of pressure and volumetric flow waveforms in the abdominal aorta are compared in Figure 2. The instantaneous FSI pressure drop between the inlet and the outlet had a maximum value of $4.2 \mathrm{mmHg}$ which was 2.62 times lower than the maximum pressure drop obtained during the CFD simulation (11 mmHg, Figure 2a). In the FSI simulation there was a clear time delay in the pressure (and flow) waves between the proximal and distal location, and the propagation speed of the pressure wave (pulse wave velocity) was estimated $4.8 \mathrm{~m} / \mathrm{s}$ (based on the foot-to-foot delay). The computed FSI outflow at the distal part of the aorta corresponded better to the measured outflow than the computed CFD flow which falsely predicted a significant amount of backflow in the end-systolic phase, followed by a close to zero outflow during diastole (Fig. 2b). At peak-systole, CFD overestimated the pressure drop (Fig. 2c) and flow velocity (Fig. 2d) compared to FSI simulations, while at end-diastole CFD simulations underestimated both pressure drop and flow velocity. The difference in pressure drop predicted by FSI and CFD was highest at the model inlet and gradually decreased towards the outlet (Fig. 2c). The velocity magnitudes predicted by FSI and CFD were equal at the model inlet (as the same velocity was imposed in both models) but at peak systole FSI predicted higher velocities than CFD towards the distal part of the aorta, while at end diastole FSI predicted lower velocities than CFD near the model outlet (Fig. 2d).

Figure 3 compares CFD and FSI simulations of velocity contours at cross sectional planes along the centerline, as well as contour plots of peak-systolic wall shear stress. As indicated in Figure 2d, CFD simulations yield much higher velocities (Fig. 3a, 3b) and shear stresses (Fig. 3c, 3d) than FSI simulations at peak systole, while CFD velocities are lower than FSI velocities at end diastole. The contour plots clearly visualize that the difference in velocity is largest in the caudal 
part of the aorta, distal to the renal arteries (Fig. 3b, 3d). In FSI simulations, zones of high wall shear stress can be found distal to the celiac bifurcation and the trifurcation, whereas zones of low wall shear stress are found cranial and lateral to where the side branches sprout (Fig. 3c). Despite the difference in absolute values, wall shear stresses simulated by CFD predict similar zones of increased or decreased WSS in the vicinity of celiac and mesenteric side branches (Fig. $3 d)$.

Figure 4 presents FSI contour plots of peak-systolic maximum principal stress at both the inner and outer surface of the aortic wall. At the inner wall, the highest maximum principal stress occurs just distal to the celiac artery, with additional peaks distal and cranial to the trifurcation (Fig. 4a). At the outer wall, the maximum principal stress concentration is highest on both lateral sides of the celiac artery and the trifurcation of mesenteric and right renal arteries, with a marked increase in stress on the left lateral side (Fig. 4b). 


\section{Discussion}

In this work a mouse-specific FSI approach has been presented to simultaneously simulate fluid dynamics and solid mechanics in the murine abdominal aorta. The abdominal aorta geometry, the flow boundary conditions as well as the material parameters for the aortic wall were obtained in vivo in the same animal. An advanced hexahedral mesh represented the geometry and the in vivo stress field was taken into account by calculating the zero-pressure geometry. Flow waveforms predicted by FSI simulations were found to correspond better to in vivo measurements than those from CFD simulations based on the same input parameters. Zones of locally decreased wall shear stress and locally increased principal stress were found near the orifice of abdominal side branches. Interestingly, these locations coincided with the regions at which a local tear in the tunica media had previously been observed in the abdominal aorta of angiotensin II-infused mice that develop dissecting abdominal aortic aneurysm.

\section{Fluid dynamics - FSI versus CFD}

Zones of locally disturbed wall shear stress in the vicinity of side branches were similarly distributed in both CFD and FSI simulations. High shear stress was observed caudal to the celiac artery and the trifurcation, while low shear stresses were found cranial and lateral to the abdominal side branches. Nevertheless, predicted flow waveforms and absolute shear stress values were significantly different between FSI and CFD simulations, especially in the distal part of the abdominal aorta, caudal to the renal arteries (Fig. 2, 3). The inclusion of distensible walls resulted in much more realistic flow velocity waveforms, with peak-systolic and enddiastolic velocities that corresponded to measured values and without backflow at early diastole (which was never observed in vivo). This is an important improvement, as it also implies that while CFD simulations indicate elevated values and oscillatory patterns in wall shear stress in 
the abdominal aorta, FSI simulations predict lower shear stress and unidirectional flow throughout the complete cardiac cycle.

In both the CFD and FSI problem, flow velocity boundary conditions were applied instead of volumetric flows. The main rationale for doing so was the fact that velocities were measured in vivo rather than flows. The velocity profiles were imposed assuming fully developed, parabolic flow in the abdominal aorta. It is important to keep in mind that Reynolds and Womersley numbers in mice are much lower than in humans $(\operatorname{Re} \cong 60, \alpha \cong 0.5$ in the abdominal aorta) which makes this a reasonable assumption, even if the descending and thoracic aorta were not included into the model [11]. Since the entrance length for such low Reynolds number is only 3.5 times the aortic diameter, we expect that the velocity profile and wall shear stresses should only be affected near the immediate inlet of the model. Since velocity profiles were imposed, the resulting volumetric inflow for the FSI case was higher than in the CFD case, which was performed with rigid walls for the image-based (end-diastolic) geometry (Fig. 2b). Despite the slightly higher imposed flow the instantaneous pressure difference over the aorta was still significantly lower in the FSI simulations due to the buffering action of the aorta that is inherently present in the FSI model. As such, less volume needs to be accelerated along the axis of the aorta, leading to less steep pressure gradients. In summary, we speculate that our mousespecific FSI approach allows for more physiologically realistic flow field predictions in the murine arterial system than the commonly used CFD approach.

\section{FSI model predictions versus PCXTM observations}


Our long-term goal is not just to demonstrate the technical feasibility and physiological superiority of mouse-specific FSI simulations, but to use these models to investigate the role of branch-related biomechanics in the initiation of cardiovascular disease in general, and dissecting aneurysm in particular. Intuitively, it makes sense that lesions would originate at the orifice of aortic side branches as these locations are subjected to local changes in anatomy (the tunica media of counts less lamellae than the aorta [3, 32]), hemodynamics (flow separation and low, oscillatory shear stresses occur near the orifice of side branches $[5,6])$ and structural mechanics (local peaks in tension arise at the branch orifice). Both in mice [33] and humans [34-36], disturbed flow patterns near side branches have been shown to be directly related to the onset of atherosclerosis. In this respect, the study of Endo et al. is particularly interesting as the authors clearly demonstrate how the abdominal branching pattern influences the local flow profiles and the onset of atherosclerotic lesions [34].

In the current manuscript we propose the hypothesis that, in the case of dissecting aneurysms, the mechanical tension near the branch orifice might play an equally important role as the locally disturbed hemodynamics. This hypothesis was initially based on observations of Gavish et al., who reported focal ruptures of the elastic lamellae in 126 of 325 investigated histological sections that included at least part of a branch orifice, but in none of the 479 sections in between branches [3]. The branch-related hypothesis was further supported by our previous work, in which we used a novel post mortem imaging technique (PCXTM) to visualize abdominal aortic aneurysms in angiotensin II-infused mice [4]. We demonstrated that the variation in aneurysm shape, ranging from Grade I (minor dilatation without thrombus) to Grade IV (polymorphic aneurysm extending into the thoracic aorta) $[37,38]$, was directly related to the number and location of focal mechanical failures of the tunica media that occurred in the vicinity of aortic 
side branches. Affected branches were (i) small suprarenal side branches, where mural ruptures led to intramural hematoma and adventitial dissection, and (ii) the celiac artery, where a focal tear led to apparent luminal dilatation. The latter tear was observed either just caudal to the celiac artery (Fig. 4d), left lateral to both celiac and mesenteric arteries (Fig. 4e) or both caudal and left lateral to the celiac artery (Fig. 4c).

In order to validate the hypothesis that branch-related ruptures initiate dissecting AAA formation in mice, longitudinal research should ideally compare early stage FSI simulations to end-stage disease location in the same mice. In the current manuscript we present a first step towards this final end since our FSI model was based on data obtained in a normal, male ApoE -- mouse. Our mouse-specific results thus provide insight into the distribution - prior to aneurysm development - of principal stresses near the orifice of abdominal side branches in general, and the celiac artery in particular. We observed that principal stresses at the inner aortic wall (Fig. 4a) were elevated just caudal to the celiac artery, while principal stresses at the outer aortic wall (Fig. 4b) were highest at the left lateral side of both celiac and mesenteric arteries. Intriguingly, these are the exact same regions at which a mural tear had previously been observed using post mortem PCXTM imaging: some animals showed a tear just caudal to the celiac (Fig. 4d), others had a large tear that ran entirely on the left lateral side of celiac and mesenteric arteries (Fig. 4e), and some animals had a tear that extended both caudal and left of the celiac artery (Fig. 4c).

These first simulation results thus seem to confirm the hypothesis that locally elevated biomechanical stresses might play an important role in the early stages of the pathophysiology of dissecting aneurysm in mice. One might therefore hypothesize that the large variation in the number and location of branch-related ruptures that was observed in previous PCXTM images 
[4] may be related to inter-mouse variations in the abdominal aortic anatomy and branching patterns. The latter hypothesis will form the basis of follow-up research.

\section{Limitations and future work}

While our preliminary results yielded interesting insights in branch-related aortic pathophysiology, they should also be interpreted with caution. For the time being our FSI simulations only represented one specific case, and the data obtained in the simulated aorta was compared to PCXTM images that were obtained in different animals. Due to the limited resolution of in vivo contrast-enhanced micro-CT imaging, smaller branches such as the intercostals and superior suprarenal side branches were not incorporated into the current model yet. While we incorporated in vivo stresses, our approach did not account for any residual stresses that might be present in the aortic tissue. Moreover, some considerations on the used boundary conditions should be taken into account when interpreting the results.

For a realistic fluid-structure interaction approach, a correct representation of the vessel wall is indispensable. Sophisticated material models and wall thickness distributions, which include the stiffening effect and the anisotropic behavior for the different layers of the arterial wall, have been described for both human [39, 40] and murine [41, 42] aortas. In the current work, however, our main interest was to capture the distension of the arterial wall that had been measured in vivo using high-frequency ultrasound (M-mode). Since a fit of complex anisotropic material models to this limited amount of in vivo data would over parameterize the problem, we sought an alternative that could describe the material with fewer parameters. The singleparameter Neo-Hookean material model was deemed unsuitable due to the lack of material stiffening at higher strains and the absence of surrounding tissue in our CSM model. A general 
fifth-order polynomial model could provide the desired material stiffening but did not solve the over parameterization. The Arruda-Boyce model, which is a reduced fifth-order polynomial model [28], was chosen since it is able to offer the desired effect of material stiffening for only two material parameters. Moreover, the model allows for a physical interpretation. Nevertheless, it remains an isotropic, hyperelastic model while actual blood vessels are complex, anisotropic structures capable of continuous and dynamical adaptation to the biomechanical environment via smooth muscle tone regulation and synthesis and organization of extracellular matrix proteins (especially elastin and collagen). At first sight, the insufficient spatial differentiation of material properties hampers unequivocal interpretation of our results, especially in the vicinity of side branches. Indeed, it is likely that the local micro-biomechanical environment near side branches has adapted and remodeled such that mechanical stress concentrations are smoothed out and accounted for by the local microstructure and organization. It is also important to take into account that the aforementioned remodeling mostly occurs in the adventitial wall, while the observed ruptures take place in the lamellae of the tunica media. But even if the actual in vivo stress concentrations are lower, our FSI simulations still provide an important insight into the geometric distribution of zones that are prone to mechano-biological remodeling, and thus might be at higher risk for mechanical perturbations.

The pulse pressure was the only variable that was not obtained in vivo in the same animal that was used for geometry and flow boundary conditions. Both invasive and non-invasive (tailcuff) pressure data that have been obtained under anesthesia showed large inter and intra mouse variability, and we judged these measurements insufficiently accurate to justify the increased suffering for the animals. The used values are representative for an anesthetized adult mouse 
[29], but evidently a change in pressure boundary conditions in combination with a similar aortic distension would affect the material stiffness and hence the magnitude of the predicted principal stresses. However, the distribution of the principal stress (and especially zones of elevated and decreased principal stress) over the aortic surface should not be affected as this is to a great extent geometry-dependent.

The presented approach will serve as a basis for later work. In future FSI simulations the in vivo geometry will be scanned with contrast-enhanced micro-CT at higher resolution to allow for inclusion of small side branches into the model. Mouse-specific pressure boundary conditions and more elaborate material models and mouse-specific wall thickness distributions will be incorporated to obtain a better prediction in the vicinity of side branches. Finally, a longitudinal approach will be followed in which baseline (prior to angiotensin II-infusion) or early stage (prior to medial tear) FSI simulations will be compared to end-stage PCXTM images in a large group of angiotensin II-infused mice. 


\section{Conclusion}

We presented mouse-specific FSI simulations yielding insight into the flow field, wall shear stress and principal stresses in the murine abdominal aorta. We observed that FSI simulations improve flow predictions compared to CFD simulations in the same animals. At the very region at which a mural tear occurs in the tunica media of angiotensin II-infused mice, principal stresses predicted by FSI were elevated in a normal $\mathrm{ApoE}^{-/-}$mouse. These preliminary results will form the basis for more elaborate research to provide deeper insights into the link between biomechanics, mechanobiology and branch-related ruptures in angiotensin-II infused mice. 


\section{Acknowledgements}

This research was funded by the Special Research Fund of Ghent University under Grant [BOF10/GOA/005] and by internal funds of the Laboratory of Hemodynamics and Cardiovascular Technology, EPFL. B.T. received a travel grant of the Flemish Fund for Scientific Research. The computational resources and services used in this work were provided by the VSC (Flemish Supercomputer Center), funded by Ghent University, the Hercules Foundation and the Flemish Government - department EWI.

\section{Conflict of Interest}

None declared. 


\section{References}

[1] Saraff, K. et al., Aortic dissection precedes formation of aneurysms and atherosclerosis in angiotensin II-infused, apolipoprotein E-deficient mice, Arteriosclerosis, Thrombosis, and Vascular Biology, 23(9): 1621-1626, 2003.

[2] Daugherty, A. et al., Angiotensin II promotes atherosclerotic lesions and aneurysms in apolipoprotein E-deficient mice, Journal of Clinical Investigation, 105(11): 1605-1612, 2000.

[3] Gavish, L. et al., Inadequate reinforcement of transmedial disruptions at branch points subtends aortic aneurysm formation in apolipoprotein-E-deficient mice, Cardiovasc Pathol, 23(3): 152-159, 2014.

[4] Trachet, B. et al., Dissecting abdominal aortic aneurysm in ang II-infused mice: suprarenal branch ruptures and apparent luminal dilatation, Cardiovascular Research, 105(2): 213$222,2015$.

[5] Trachet, B. et al., Longitudinal follow-up of ascending versus abdominal aortic aneurysm formation in angiotensin II-infused ApoE-/- mice, Artery research, 8(1): 16-23, 2014.

[6] Trachet, B. et al., An integrated framework to quantitatively link mouse-specific hemodynamics to aneurysm formation in angiotensin II-infused ApoE -/- mice, Annals of Biomedical Engineering, 39(9): 2430-44, 2011.

[7] Greve, J.M. et al., Allometric scaling of wall shear stress from mice to humans: quantification using cine phase-contrast MRI and computational fluid dynamics, American Journal of Physiology-Heart and Circulatory Physiology, 291(4): H1700H1708, 2006.

[8] Feintuch, A. et al., Hemodynamics in the mouse aortic arch as assessed by MRI, ultrasound, and numerical modeling, American Journal of Physiology-Heart and Circulatory Physiology, 292(2): H884-H892, 2007.

[9] Van Doormaal, M. et al., Inputs for Subject-Specific Computational Fluid Dynamics Simulation of Blood Flow in the Mouse Aorta, Journal of Biomechanical Engineering, 136(10): 101008-101008, 2014.

[10] Hoi, Y. et al., Correlation Between Local Hemodynamics and Lesion Distribution in a Novel Aortic Regurgitation Murine Model of Atherosclerosis, Annals of Biomedical Engineering, 39(5): 1414-1422, 2011.

[11] Trachet, B. et al., The Impact of Simplified Boundary Conditions and Aortic Arch Inclusion on CFD Simulations in the Mouse Aorta: A Comparison With Mouse-specific Reference Data, Journal of Biomechanical Engineering, 133(12): 121006-13, 2011.

[12] Huo, Y.L. et al., The flow field along the entire length of mouse aorta and primary branches, Annals of Biomedical Engineering, 36(5): 685-699, 2008.

[13] Ford, M.D. et al., Hemodynamics of the Mouse Abdominal Aortic Aneurysm, Journal of Biomechanical Engineering, 133(12), 2011.

[14] Bols, J. et al., A computational method to assess the in vivo stresses and unloaded configuration of patient-specific blood vessels, J Comput Appl Math, 246(0): 10-17, 2013.

[15] Bols, J. et al., Inverse modelling of image-based patient-specific blood vessels: zero pressure geometry and in vivo stress incorporation, Esaim - Math Model Num Anal, 47(4): 1059-1075, 2013. 
[16] Broisat, A. et al., Assessing Low Levels of Mechanical Stress in Aortic Atherosclerotic Lesions From Apolipoprotein E-/- Mice-Brief Report, Arteriosclerosis, Thrombosis, and Vascular Biology, 31(5): 1007-1010, 2011.

[17] Reymond, P. et al., Physiological simulation of blood flow in the aorta: Comparison of hemodynamic indices as predicted by 3-D FSI, 3-D rigid wall and 1-D models, Medical Engineering \& Physics, 35(6): 784-791, 2013.

[18] Rissland, P. et al., Abdominal aortic aneurysm risk of rupture: patient-specific FSI simulations using anisotropic model, Journal of Biomechanical Engineering, 131(3): 031001, 2009.

[19] Scotti, C.M. et al., Wall stress and flow dynamics in abdominal aortic aneurysms: finite element analysis vs. fluid-structure interaction, Comput Methods Biomech Biomed Engin, 11(3): 301-22, 2008.

[20] Degroote, J. et al., Stability of a coupling technique for partitioned solvers in FSI applications, Computers \& Structures, 86(23-24): 2224-2234, 2008.

[21] Leung, J.H. et al., Fluid structure interaction of patient specific abdominal aortic aneurysms: a comparison with solid stress models, Biomed Eng Online, 5: 33, 2006.

[22] Chandra, S. et al., Fluid-Structure Interaction Modeling of Abdominal Aortic Aneurysms: The Impact of Patient-Specific Inflow Conditions and Fluid/Solid Coupling, Journal of Biomechanical Engineering, 135(8): 081001-081001, 2013.

[23] Rabben, S.I. et al., Ultrasound-based vessel wall tracking: an auto-correlation technique with RF center frequency estimation, Ultrasound in Medicine \& Biology, 28(4): 507517, 2002.

[24] Bols, J. et al., Unstructured hexahedral mesh generation in complex vascular structures using a grid-based approach, Computer Methods in Biomechanics and Biomedical Engineering, in press, 2014.

[25] Westerhof, N. et al., An artificial arterial system for pumping hearts. Vol. 31. 1971. 776781.

[26] Thompson, S. et al., Hereditary differences in serum proteins of normal mice Proceedings of the Society for Experimental Biology and Medicine, 87(2): 315-317, 1954.

[27] Chen, G. et al., Impaired erythrocyte deformability in transgenic HO-1G143H mutant mice, Transgenic Research, 24(1): 173-178, 2015.

[28] Arruda, E.M. et al., A 3-dimensionl constitutive model for the large stretch behavior of rubber elastic materials, Journal of the Mechanics and Physics of Solids, 41(2): 389412, 1993.

[29] Reddy, A.K. et al., Measurement of aortic input impedance in mice: effects of age on aortic stiffness. Vol. 285. 2003. H1464-H1470.

[30] Degroote, J. et al., Performance of a new partitioned procedure versus a monolithic procedure in fluid-structure interaction, Computers \& Structures, 87(11-12): 793-801, 2009.

[31] Degroote, J. (2010), Development of Algorithms for the Partitioned Simulation of Strongly Coupled Fluid-structure Interaction Problems, in Faculty of EngineeringGhent University: Ghent. p. 108-110.

[32] Gavish, L. et al., Low level laser arrests abdominal aortic aneurysm by collagen matrix reinforcement in apolipoprotein E-deficient mice, Lasers in Surgery and Medicine, 44(8): 664-674, 2012.

[33] Cheng, C. et al., Atherosclerotic Lesion Size and Vulnerability Are Determined by Patterns of Fluid Shear Stress, Circulation, 113(23): 2744-2753, 2006. 
[34] Endo, S. et al., Flow patterns and preferred sites of atherosclerotic lesions in the human aorta - II. Abdominal aorta, Biorheology, 51(4): 257-274, 2014.

[35] Caro, C.G., Discovery of the role of wall shear in atherosclerosis, Arteriosclerosis, Thrombosis, and Vascular Biology, 29(2): 158-61, 2009.

[36] Ku, D.N. et al., Pulsatile flow and atherosclerosis in the human carotid bifurcation positive correlation between plaque location and low and oscillating shear-stress, Arteriosclerosis, 5(3): 293-302, 1985.

[37] Manning, M.W. et al., Abdominal aortic aneurysms: fresh insights from a novel animal model of the disease, Vascular Medicine, 7(1): 45-54, 2002.

[38] Daugherty, A. et al., Antagonism of AT2 receptors augments Angiotensin II-induced abdominal aortic aneurysms and atherosclerosis, British Journal of Pharmacology, 134(4): 865-870, 2001.

[39] Gasser, T.C. et al., A rate-independent elastoplastic constitutive model for biological fiberreinforced composites at finite strains: continuum basis, algorithmic formulation and finite element implementation, Computational Mechanics, 29(4-5): 340-360, 2002.

[40] Holzapfel, G.A. et al., A new constitutive framework for arterial wall mechanics and a comparative study of material models, Journal of Elasticity, 61(1-3): 1-48, 2000.

[41] Bersi, M.R. et al., Disparate Changes in the Mechanical Properties of Murine Carotid Arteries and Aorta in Response to Chronic Infusion of Angiotensin-II, International journal of advances in engineering sciences and applied mathematics, 4(4): 228-240, 2013.

[42] Collins, M.J. et al., Mechanical properties of suprarenal and infrarenal abdominal aorta: Implications for mouse models of aneurysms, Medical Engineering \& Physics, 33(10): 1262-1269, 2011. 


\section{Figures}

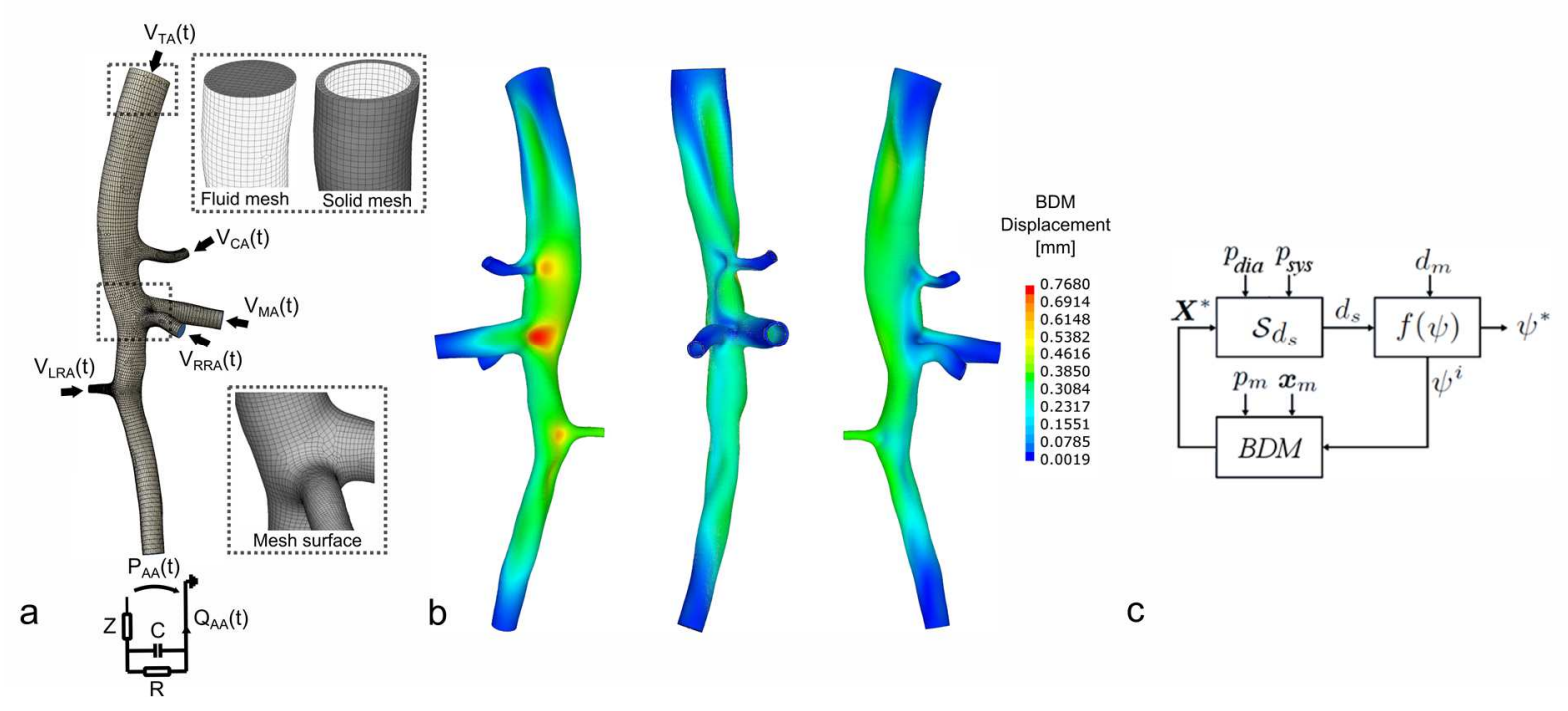

Figure 1. FSI methodology. a. Fluid domain and CFD boundary conditions: a velocity inlet at the thoracic artery (TA), velocity outlets at the celiac artery (CA), right renal artery (RRA), mesenteric artery (MA) and left renal artery (LRA) and a windkessel pressure outlet at the distal abdominal aorta (AA). b. Zero-pressure geometry (for three different views) computed using the BDM (backward displacement method), with contour plots of the displacement denoting the distance between the zero-pressure geometry and the image-based geometry. c. Schematic representation of the BDM-based material parameter optimization framework (BDMPO). $\mathrm{x}_{\mathrm{m}}$ denotes the in vivo geometry, $\mathrm{p}_{\mathrm{m}}$ the internal pressure load, $\mathrm{X}^{*}$ the geometry after a BDM iteration, $\mathrm{S}$ the inflated geometry after a static CSM simulation starting from $\mathrm{X}^{*}, \mathrm{P}_{\mathrm{dia}}$ the enddiastolic state, $P_{\text {sys }}$ the peak-systolic state, $d_{s}$ the simulated distension, $d_{m}$ the measured distension and $\mathrm{f}(\boldsymbol{\psi})$ the objective function to be minimized (eq. 2 ). 

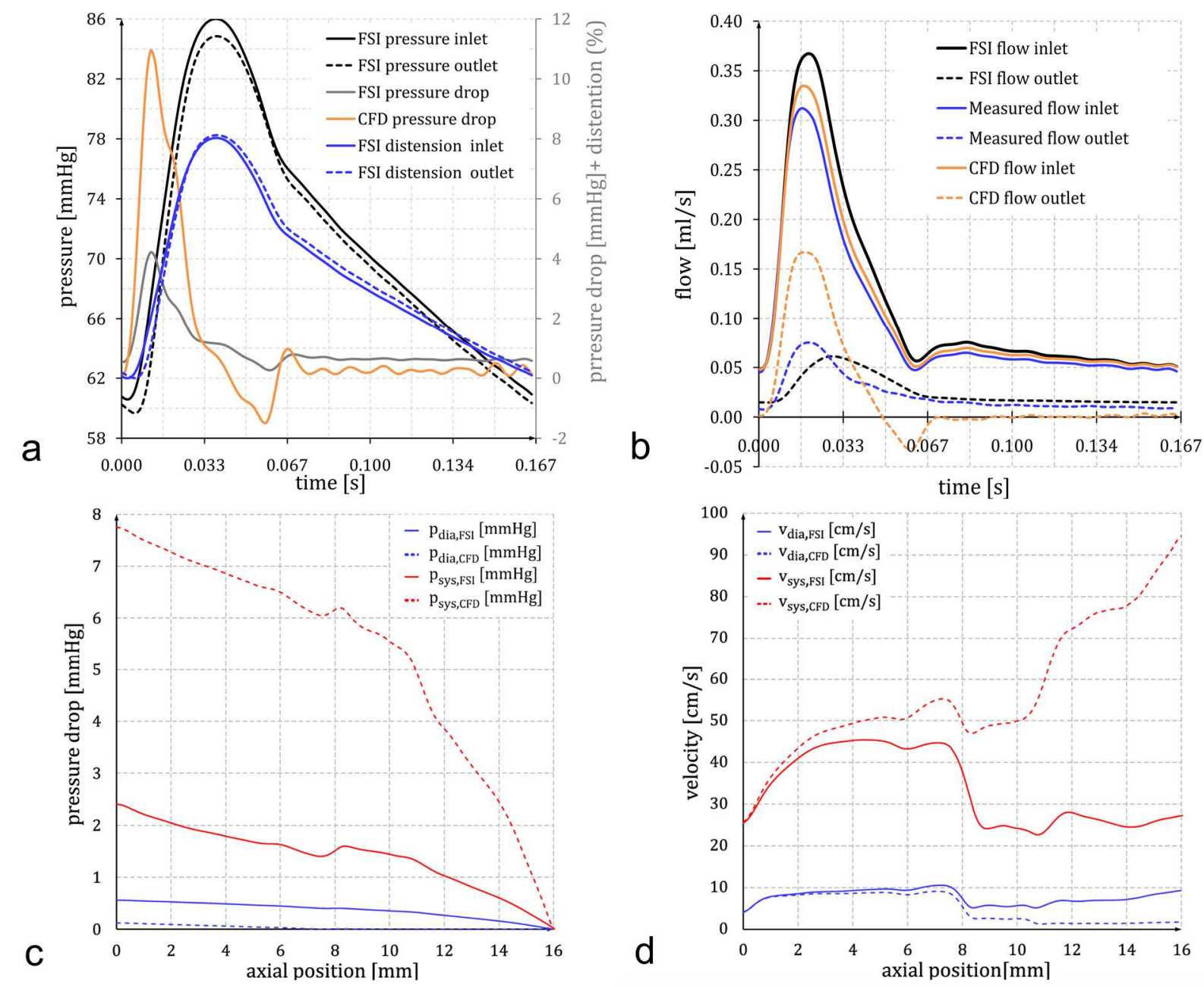

Figure 2. Quantitative comparison of hemodynamics between FSI and CFD. a. Evolution of pressure (FSI), pressure drop (CFD + FSI) and distension (FSI) as a function of time. Waveforms are shown at the inlet (TA) and outlet (AA) of the aorta. b. Evolution of flow rate (CFD+FSI+in vivo measurements) as a function of time. Waveforms are shown at the inlet (TA) and outlet (AA) of the aorta. c. Evolution of pressure drop (CFD + FSI) as a function of axial centerline position along the aorta. Waveforms are show at peak-systole (in red) and enddiastole (in blue). d. Evolution of velocity magnitude (CFD + FSI) as a function of axial centerline position along the aorta. Waveforms are shown at peak-systole (in red) and enddiastole (in blue). 

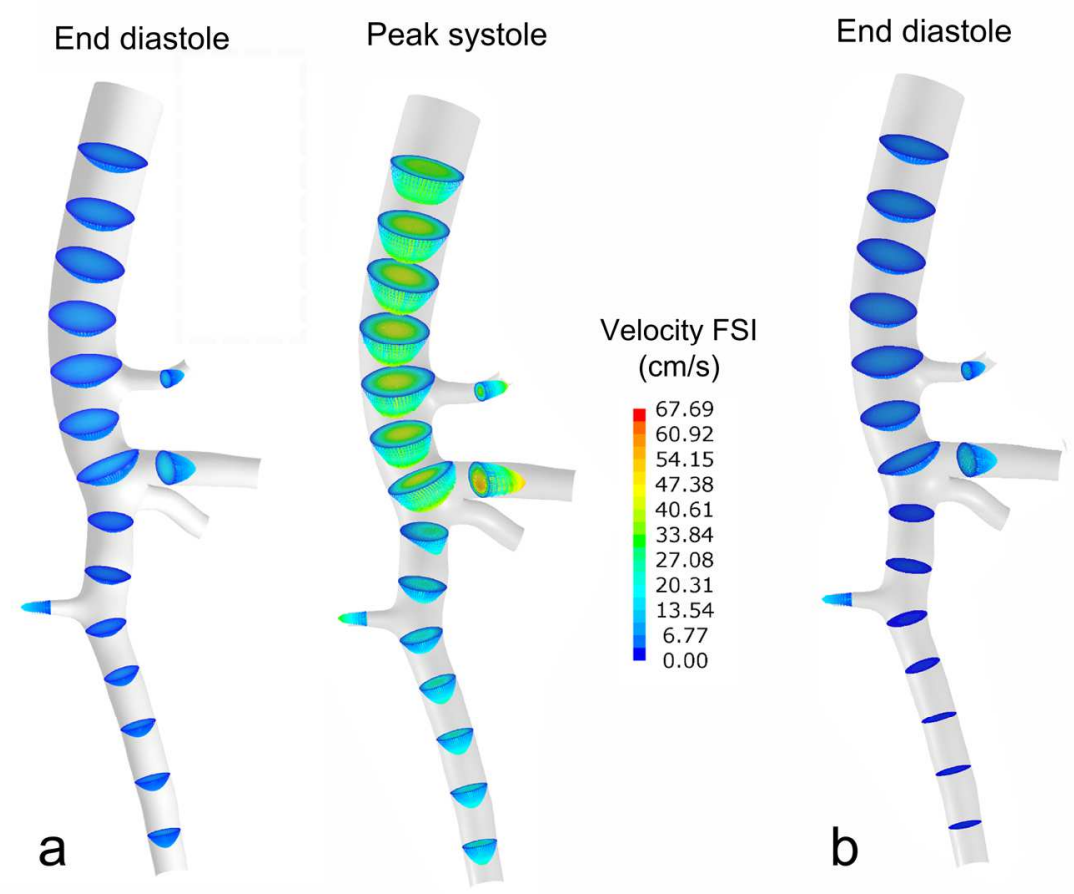

Peak systole
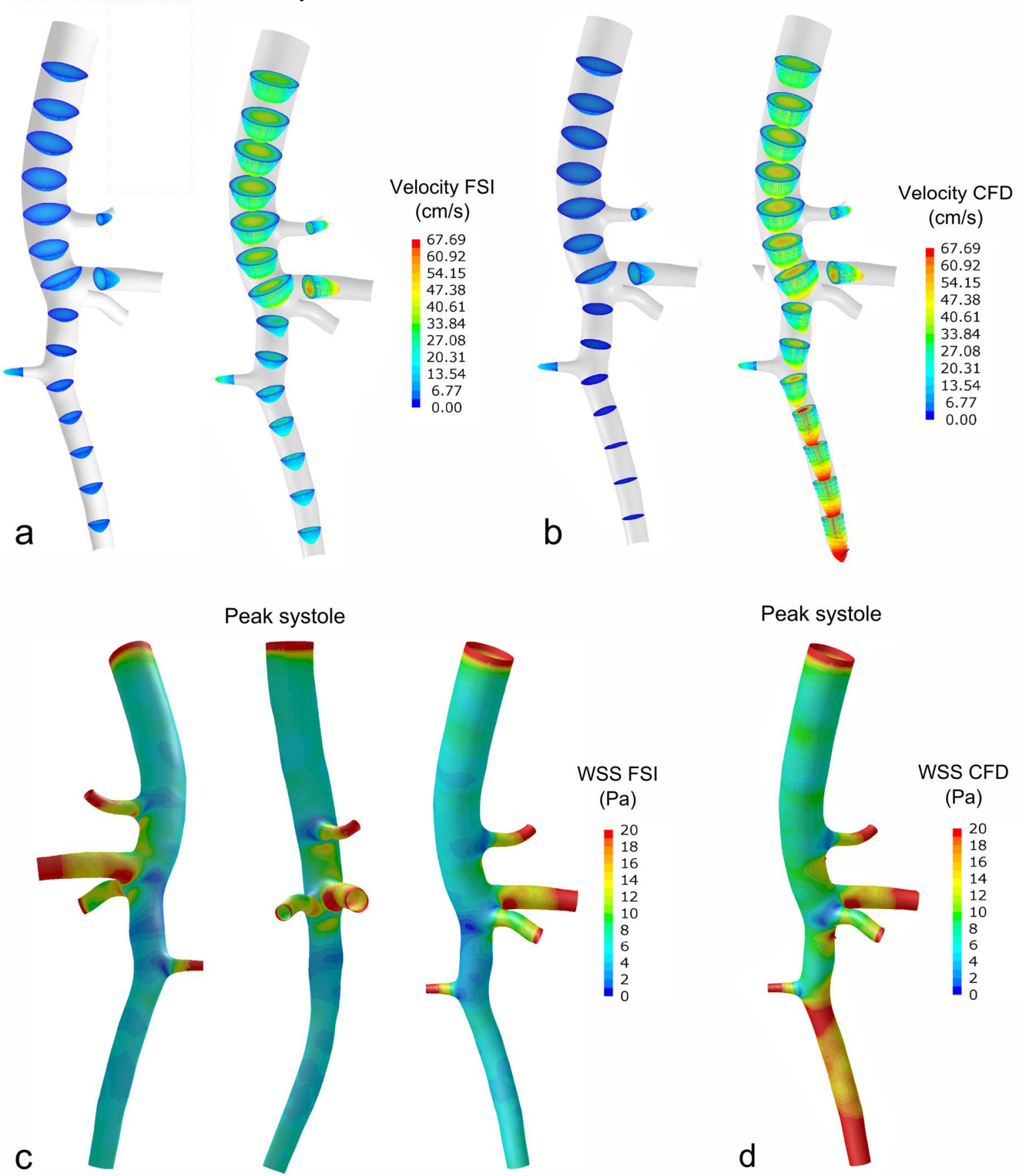
Figure 3. Qualitative comparison of velocity and wall shear stress between FSI and CFD. a. FSI velocity contours colored by magnitude at cross-sectional planes along the centerline at the moment of end-diastole (left) and peak-systole (right). b. CFD velocity contours colored by magnitude at cross-sectional planes along the centerline at the moment of end-diastole (left) and peak-systole (right). c. FSI contour plots of peak-systolic wall shear stress distribution, for three different views. d. CFD contour plots of peak-systolic wall shear stress distribution. 


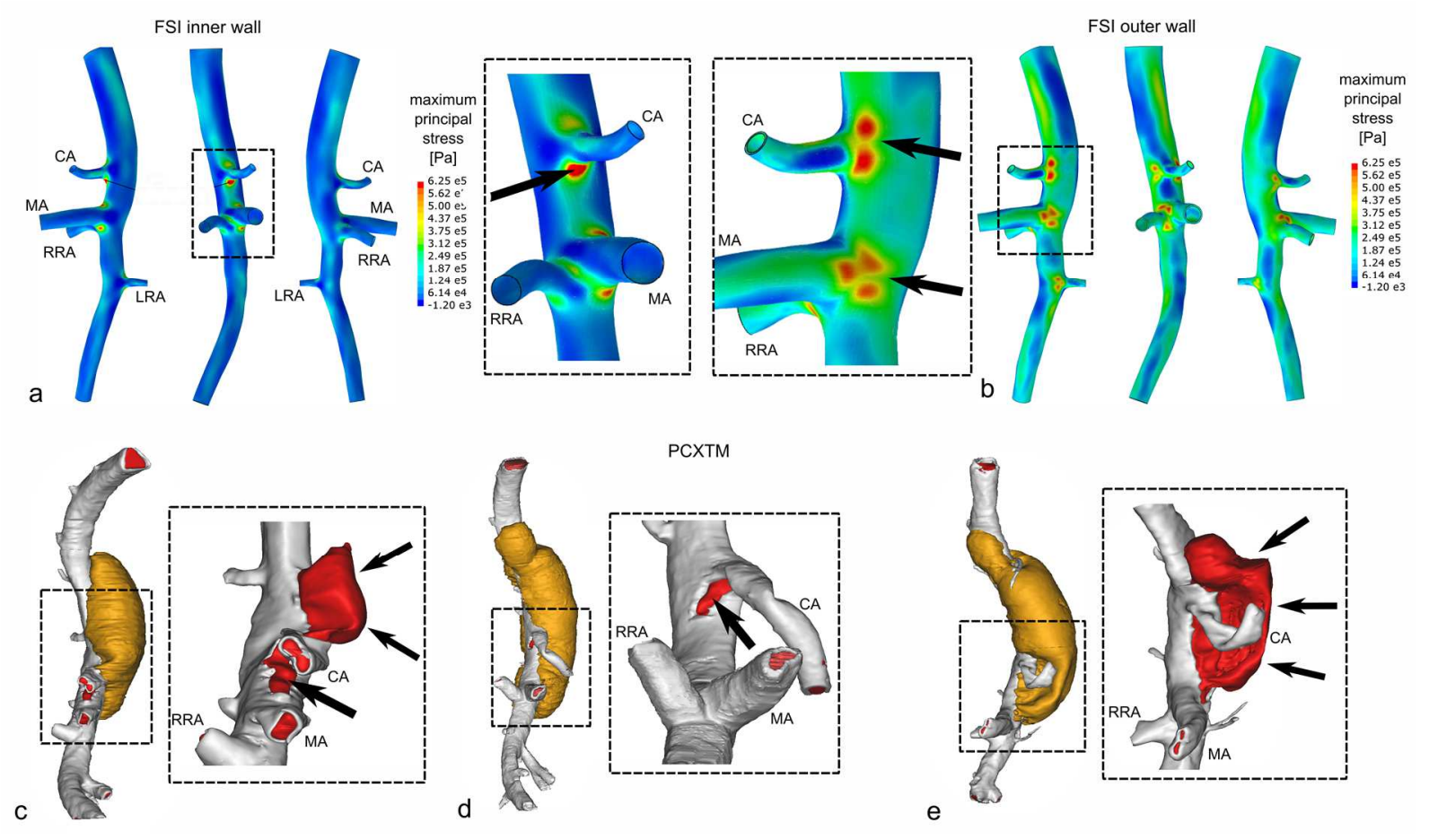

Figure 4. Comparison between FSI results and location of medial tear in PCXTM images. FSI contour plots (panels a-c) are shown at peak-systole, for three different views. PCXTM images (panels e-f) are shown as 3D representations of dissecting aneurysms in 3 different animals, reproduced from [4]. The blood-filled lumen is shown in red, tunica media in white and the intramural hematoma in orange. a. Contour plots of the maximum principal stress at the inner wall (i.e. at the fluid-structure interface). Arrow indicates peak stress caudal to the celiac artery. b. Contour plots of the maximum principal stress at the outer wall. Arrows indicate peak stress on the left lateral side of the celiac and mesenteric artery. c. PCXTM image showing a large tear in the tunica media, caudal as well as left lateral to the celiac artery. d. PCXTM image showing a small tear in the tunica media, caudal to the celiac artery. e. PCXTM image showing a large tear in the tunica media, left lateral from the celiac to the mesenteric artery. 